\title{
Early Outcomes of Emergency Surgery for Left-Sided Mechanical Valve Thrombosis
}

\author{
Hesham Zayed Saleh, MD, FRCS-CTh, Mohamed Azzam, MD, Ahmed AH Swailum, MD, \\ Alaa Farouk, MD \\ Department of Cardiothoracic Surgery, Cairo University, Cairo, Egypt
}

\section{ABSTRACT}

Background: Valve thrombosis is a potentially lethal complication of mechanical cardiac valves. We examined the clinical characteristics as well as the early outcomes of patients undergoing emergency surgery for left-sided mechanical valve thrombosis.

Methods: Between January 2012 and May 2020, 104 consecutive patients were offered an emergency redo surgery for acute mechanical valve thrombosis. Ninety-seven of these patients were included in the current study.

Results: The mean age was $34.2 \pm 10.3$ years. Most of the patients were females (61 patients), and 27 patients $(27.8 \%)$ were pregnant. The mitral valve was the site of thrombosis in 81 patients. Inadequate anticoagulation was found in $60.8 \%$ of patients. The overall early mortality was $32.9 \%$ (32 patients) with an operative mortality of $25.7 \%$. Outcomes in the pregnant subgroup tended to be worst with a maternal mortality in the range of $37 \%$, and with fetal and neonatal survival as low as $33.3 \%$.

Conclusion: The overall mortality in cases of mechanical valve thrombosis warranting surgery remains high. Since inadequate anticoagulation seems to be one of the major precipitating factors, the current study highlights the need for improvements in anticoagulation practices. The use of tissue valves should also be contemplated more seriously in some younger patients, especially females expressing the desire for future pregnancies.

\section{INTRODUCTION}

Contrary to the practice in Western countries, rheumatic valve disease continues to be quite prevalent in Egypt [El-Aroussy 2013] and remains the major indication for valvular surgery in developing countries [Soler-Soler 2000]. Given the rheumatic pathology that often is not amenable to repair and the associated younger age of the patients, the use of mechanical valves is commonplace.

Mechanical valve thrombosis (MVT) is one of the most dreaded complications of the use of mechanical heart valves.

Received September 14, 2021; accepted September 23, 2021.

Correspondence: Hesham Zayed Saleh, Assistant Professor of Cardiothoracic Surgery, Faculty of Medicine, Cairo University, Kasr Al Ainy, Cairo University Hospitals, Department of Cardiothoracic Surgery, 14113; (e-mail: hesham.z.saleb@kasralainy.edu.eg).
Probably due to a combination of lower levels of education and compliance to anticoagulation [Sharaf 2017], the reported incidence of such a fatal complication is as high as $6.1 \%$ per patient-year in developing countries compared with a much lower incidence of $0.3-1.3 \%$ per patient-year in the Western world [Nishimura 2014].

Most of the published reports about MVT included heterogeneous groups of patients undergoing surgery with variable degrees of urgency [Durrleman 2004; Ahn 2008; Ma 2015; Roudaut 2003; Hassouna 2020]. Older reports including only patients who underwent emergency surgery included mostly older generations of mechanical valves like tilting disc and ball \& cage valves, apart from reporting from a different clinical setup where mechanical valves are less commonly used [Buttard 1997]. With such heterogeneity in the available literature, examining the characteristics and outcomes of patients undergoing emergency surgery for MVT in a developing country setting in the current era is worthy.

The purpose of the current study is to examine the clinical characteristics as well as the early outcomes of patients undergoing emergency surgery for left-sided MVT in the setting of an Egyptian University hospital.

\section{PATIENTS AND METHODS}

This is a retrospective observational study including patients who received an emergency redo operation for acute MVT in the cardiothoracic surgery department at Cairo University Hospitals. During the period between January 2012 and May 2020, 104 consecutive patients were offered an emergency redo surgery after the diagnosis of acute MVT. Seven patients were excluded, six of them due to deficient files and one patient for being reoperated for a mechanical tricuspid prosthesis. The remaining 97 patients were included in the study, and their individual files were reviewed to extract clinical data. The study was approved by the institutional ethical committee, and given the retrospective nature of the study, patient consent was waived.

Once the diagnosis of MVT was suspected, patients were started on intravenous unfractionated heparin. Transthoracic echocardiography (TTE) was done in all patients. It was deemed diagnostic of valve thrombosis when transvalvular gradients of the mechanical prosthesis in question were elevated along with dysfunction of a mobile element of the prosthesis in the presence of thrombus echo density. Fluoroscopy was performed in 85 patients $(87.6 \%)$ and TEE in 16 patients $(16.4 \%)$ to confirm the diagnosis. 
Table 1. Preoperative patients' characteristics

\begin{tabular}{|c|c|}
\hline Variable & Value \\
\hline Age (years) & $34.2 \pm 10.3$ \\
\hline Female & $61(62.8 \%)$ \\
\hline Pregnancy & $27(27.8 \%)$ \\
\hline \multicolumn{2}{|l|}{ Primary valvular implants: } \\
\hline MVR & $77(79.3 \%)$ \\
\hline AVR & $12(12.3 \%)$ \\
\hline DVR & $5(5.1 \%)$ \\
\hline Bentall & $3(3 \%)$ \\
\hline Time from initial operation & 2 weeks - 189 months \\
\hline \multicolumn{2}{|l|}{ Initial presentation: } \\
\hline Worsening dyspnea & $83(85.5 \%)$ \\
\hline Embolic events & $11(11.3 \%)$ \\
\hline Others & $2(2 \%)$ \\
\hline \multicolumn{2}{|l|}{ NYHA Class: } \\
\hline NYHA Class III & $5(5.1 \%)$ \\
\hline NYHA Class IV & $78(80.4 \%)$ \\
\hline Preoperative mechanical ventilation & $11(11.3 \%)$ \\
\hline Creatinine & $1.1 \pm 0.4$ \\
\hline EF & $51.7 \pm 9.4$ \\
\hline Atrial fibrillation & $48(49.4 \%)$ \\
\hline Pulmonary artery pressure & $52.7 \pm 16.8$ \\
\hline Left atrial diameter & $5.2 \pm 1.2$ \\
\hline
\end{tabular}

Upon confirmation of the diagnosis of MVT, patients were referred for emergency surgery if considered inappropriate candidates for thrombolytic therapy by the cardiology department. In only six patients, surgery was preceded by a trial of thrombolysis that was abandoned, due to worsening hemodynamics. An International Normalized Ratio (INR) was obtained at the time of hospitalization in all patients. A value of less than 2 was used to define inadequate anticoagulation.

\section{Surgical techniques}

All reoperations were done through median sternotomy. Aortic and right atrial or bicaval cannulation was done, depending on the valve being re-replaced. Femoral cannulation was resorted to in eight patients. In six patients that was due to hemodynamic instability, one due to cardiac arrest on induction of anesthesia, in another patient that was due to massive bleeding on sternal re-entry. All operations in non-pregnant patients were conducted under moderate hypothermia with myocardial protection using cold potassium cardioplegia and topical ice slush. All patients had the culprit valve replaced with mechanical bileaflet prosthesis except for two patients who received a bioprosthesis on account of age.
Table 2. Anticoagulation status

\begin{tabular}{ll}
\hline & $\mathrm{N}(\%)$ \\
\hline Inadequate anticoagulation (INR < 2 on admission) & $59(60.8 \%)$ \\
Anticoagulation stopped without medical advice & $18(18.5 \%)$ \\
Regular INR checks after initial valve replacement & $51(52.5 \%)$ \\
Recent switch to heparin & $24(24.7 \%)$
\end{tabular}

\section{Management of pregnant patients}

Female patients of childbearing age were assessed for pregnancy state and pregnant cases received immediate obstetric consultation and co-management. In case of pregnancy, assessment of fetal viability was undertaken before and after emergency surgery. In case of non-viability, evacuation was performed postoperatively within the same hospital stay after attaining hemodynamic stability. In case of pregnancy of 30 weeks or more, delivery by caesarian section was performed in the cardiac surgery operating room prior to sternotomy with closure of the abdominal wound after protamine administration at the conclusion of the cardiac procedure. Fetal outcomes also were reported.

Cardiac operations were conducted under normothermia with increased CPB flows to maintain blood pressure above $70 \mathrm{mmHg}$ throughout the procedure.

While reporting the data, continuous variables were expressed as the mean \pm standard deviation $(\mathrm{SD})$ while categorical variables were expressed as number and frequencies $(\%)$.

\section{RESULTS}

Sixty-two of the 97 patients $(60.1 \%)$ had received their initial valve replacement in another center, making most of the clinical data surrounding their initial valve replacement unavailable. Clinical characteristics of the 97 patients are summarized in Table 1. (Table 1) There were 61 females (62.8\%) and 36 males with a mean age of $34.2 \pm 10.3$. The interval between initial valve replacement and presentation ranged between 15 days and 189 months. Thrombosis occurred in the mitral position in the majority of cases $(83.5 \%)$, and in the aortic position in only 16 patients. Three of the patients having thrombosis in the aortic position had had a previous Bentall procedure.

The most common initial symptom was a worsening dyspnea occurring in $85.5 \%$ of the cases. In two cases, the presentation was a rapidly evolving shock over a few hours. Of note was the sudden development of severe hemodynamic instability in five patients who initially were in NYHA class III at the time of admission. Of the patients, $11.3 \%$ required preoperative mechanical ventilation. Of the 11 patients presenting with embolic events, there were five strokes, three cases of transient ischemic attacks, two cases with myocardial infarction in the right coronary artery territory, and one case of lower limb ischemia requiring embolectomy. 
Table 3. Operative findings and procedure

\begin{tabular}{lc}
\hline & Value \\
\hline Pannus + Thrombus & $15(15.4 \%)$ \\
Surgical procedure: & \\
MVR & $76(78.3 \%)$ \\
AVR & $14(14.4 \%)$ \\
Aortic x-clamp time (min) & $91.4 \pm 21.2$ \\
CPB time (min) & $122.1 \pm 39.7$ \\
Adjunctive procedures & \\
Tricuspid repair & $11(11.3 \%)$ \\
LAA closure & $43(44.3 \%)$
\end{tabular}

Table 2 shows the anticoagulation status of patients on admission. (Table 2) In $60.8 \%$ of the patients, INR levels denoted inadequate anticoagulation. Of the patients, $18.5 \%$ had stopped anticoagulation altogether without medical advice, and only $52.5 \%$ of the patients followed up their anticoagulation status with regular INR check ups.

In the pregnant subgroup of patients $(\mathrm{N}=27)$, the majority (13 patients, $48.1 \%$ ) was on low-molecular weight heparin $(\mathrm{LMWH})$. Seven patients $(25.9 \%)$ were on unfractionated heparin (UFH). Five patients were on warfarin (18.5\%), while two patients $(7.4 \%)$ had stopped warfarin without any substitution or medical advice.

Time from hospital admission to operation ranged between four and 21 hours.

Table 3 outlines the operative procedures and operative findings (Table 3). A pannus was identified in 15 patients. Table 4 outlines the early outcomes in these patients. The overall early mortality was $32.9 \%$ (32 patients), with an operative mortality of $25.7 \%$. Seven patients expired preoperatively while being prepared for surgery. There were four intraoperative deaths $(4.1 \%)$, due to inability to wean off cardiopulmonary bypass. Multi-organ failure was the most common cause of death in the postoperative period occurring in 18 patients (18.5\%). Less common causes of early mortality in the postoperative period included one case of massive stroke and one case of vasoplegia syndrome.

Early mortality was somehow higher in the pregnant subgroup, when compared with the non-pregnant patients. Out of 27 pregnant patients, there were 10 early mortalities (37\%), one occurred preoperatively. Figure 1 outlines the fetal and neonatal outcomes. Two fetuses (7.4\%) were already non-viable on admission preoperatively. Only two neonates had attained full-term at the time of surgery and did well until discharge from the hospital. Eight neonates were delivered preterm, with only three surviving. In total, the fetal and neonatal survival perioperatively was low $(33.3 \%)$.

\section{DISCUSSION}

Although the early mortality rate in our series (32.9\%) with an operative mortality of $25.7 \%$ seems elevated, it
Table 4. Early outcomes

\begin{tabular}{lc}
\hline & Value \\
\hline Early mortality & $32(32.9 \%)$ \\
Renal failure & $8(8.2 \%)$ \\
Re-exploration for bleeding & $9(9.2 \%)$ \\
Deep wound infection & $3(3 \%)$
\end{tabular}

remains comparable to many published series with an early overall mortality ranging between $28.6 \%$ and $41.3 \%$ in patients admitted with a diagnosis of MVT [Durrleman 2004; Ma 2015; Buttard 1997]. The advanced stage in our patient group is evident by the high percentage of patients in NYHA class IV $(80.4 \%)$, the percentage of patients requiring preoperative ventilation $(11.3 \%)$, and the fact that they were not considered candidates for thrombolysis due to a high thrombotic burden. Other authors reporting better survival rates not only included more hemodynamically stable patients, but also reported only operative mortality rather than total inhospital mortality [Ahn 2008; Hassouna 2020].

The striking finding in our patients, was that $60.8 \%$ of them were inadequately anticoagulated on admission and only $52.5 \%$ reported regular follow up of their anticoagulation status after their initial valve replacement. Given that most of these patients received their initial operations in other centers, it was difficult to access their anticoagulation clinics data. Such alarming findings highlight how challenging maintenance of long-term adequate anticoagulation can be, especially in developing countries. Using a more stringent definition than the one we used (INR less than 2.5 compared with INR of 2 in the current study), Durrleman et al reported inadequate oral anticoagulation found at the time of diagnosis in $54 \%$ of their patients, with $26 \%$ of those attributed to poor patient compliance [Durrleman 2004]. It is worth remembering that such figures were obtained in a western country practice, where long-term anticoagulation monitoring tends to be more efficient. In developing countries, the situation tends to be worse. Previous work from our institution showed that only $42.7 \%$ of patients had satisfactory adherence levels to anticoagulation after mechanical valve implantation [Sharaf 2017]. Al Halees reported that only $25 \%$ of patients attending their anticoagulation clinics in Saudi Arabia fell within the target INR range, citing regions where even access to warfarin was a problem [Al Halees 2007]. In another study from South Africa, most patients' anticoagulation following implantation of a mechanical valve was judged unsatisfactory [Williams 2004]. The problematic nature of long-term anticoagulation in developing countries led some authors to argue in favor of more liberal use of bioprosthesis in younger patients [Al Halees 2007; Choudhary 2016]. As such debates continue, it seems there remains plenty of work to be done to improve current anticoagulation practices in developing countries.

Another important finding in the current study was the fact that sudden and severe hemodynamic deterioration occurred 


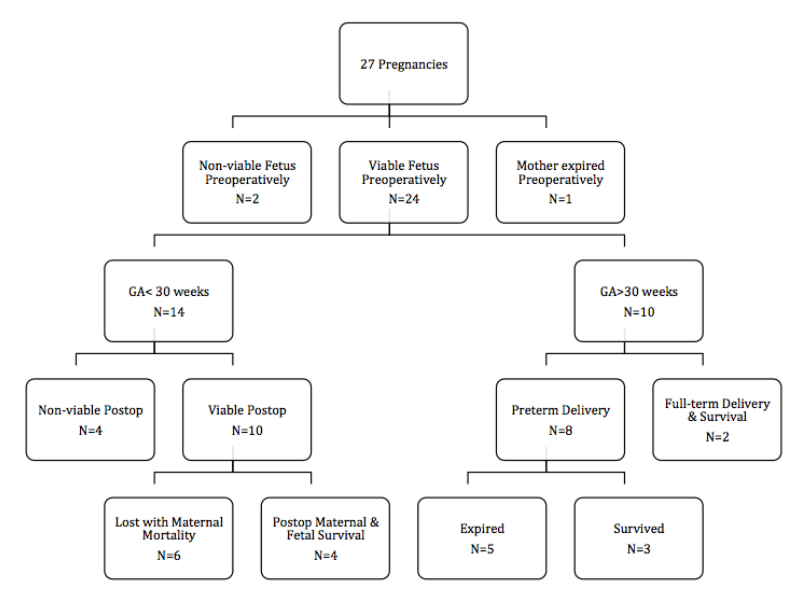

Figure 1. Non-viable Fetus Preoperatively: $\mathrm{N}=2$

Viable Fetus preoperatively; $\mathrm{N}=24$

shortly after admission of seemingly stable patients in $5.1 \%$ of the cases. This strengthens the notion that surgery should be offered emergently once indicated for MVT, even if patients seem reasonably stable. Such a practice was recommended by other groups [Durrleman 2004] and would probably complement previous studies showing longer duration of symptoms to be associated with higher mortality thus also warranting earlier referral to specialized centers [Tsai 1993].

Contrary to the practice of many other authors [Durrleman 2004; Roudaut 2003], our practice is to rereplace any mechanical valve once MVT is diagnosed without opting for thrombectomy in any case. Previously, other authors found thrombectomy to be associated with a significantly higher incidence of re-thrombosis during follow up [Martinell 1991]. In accordance with intra-operative observations, animal studies showed thrombotic material to mainly be around the hinges in bileaflet mechanical valves [Meuris 2005]. Furthermore, another animal study using scanning electron microscopy found large amounts of calcium and phosphate deposits on the hinge areas in explanted valves [Yin 2006]. Given these findings, we believe complete clearance of thrombosis apart from being impossible to ascertain, also carries the risk of damage to the valve hinge structures.

Given that almost one-third of our patients (27.8\%) were pregnant females, this subgroup was further examined separately. The hypercoagulable state of pregnancy coupled with the necessary more complicated management of anticoagulation is known to be associated with an increased risk both to the mother and fetus [Sillesen 2011; Van Hagen 2015]. A study from the UK showed that only $28 \%$ of the women with a prosthetic valve had a good maternal and fetal outcome [Vause 2017]. Despite recent guidelines recommending oral anticoagulation under strict follow up for at least the second and third trimester [Regitz-Zagrosek 2018], most of our patients, regardless of gestational age (74\%), were on heparin often in sub-therapeutic doses without reporting any adequate laboratory follow up. Such findings were reported by other working groups in the country. Elsayed et al reported on 16 pregnant patients presenting with PVT with the majority (12 out of 16 patients) being either on LMWH or UFH, often in sub-therapeutic doses [Elsayed 2019]. The results of surgical intervention in their group of patients were comparable with ours, as they reported an early maternal mortality rate of $37.5 \%$ and a favorable fetal outcome in only $31.2 \%$ of the cases. Elassy et al. reported much more favorable outcomes in a pregnant group of 23 patients with a maternal mortality of $8.7 \%$ and 13 surviving babies. However, $26 \%$ of these patients presented with native valve lesions to undergo first time rather than redo surgery, $40 \%$ of their patients underwent surgery on the nearest elective operative slot, and the more hemodynamically stable profile in most of the cases is evident by the fact that $13 \%$ of them were being allowed vaginal delivery [Elassy 2014]. With many authors reporting fewer complications and lesser rates of fetal loss in pregnant patients with tissue valves when compared with mechanical valves [Heuvelman 2013; Lawley 2014] and with near perfect anticoagulation seeming difficult to achieve in our current settings, the option of using tissue valves in young women contemplating future pregnancies should be given more weight while counseling prior to surgery.

The limitations of the current study include its retrospective nature and the fact that it included only patients with a definite diagnosis, who underwent surgery in a tertiary referral center, which may have introduced some sampling bias. The relatively limited number of patients over a relatively long period is another limitation although to the best of our knowledge this case series represents one of the largest in the English literature. The fact that most of the patients included had received their initial valve replacement in other centers made the data surrounding their initial valvular procedures and follow up unavailable.

\section{CONCLUSION}

The overall mortality in cases of MVT warranting surgery remains high. Since inadequate anticoagulation seems to be one of the major precipitating factors, the current study highlights the need for improvements in anticoagulation practices nationwide. The use of tissue valves should also be contemplated more seriously in some younger patients, especially females expressing the desire for future pregnancies.

\section{REFERENCES}

Ahn H, Kim K, Kim CK, Kim CY. 2008. Surgical management of mechanical valve thrombosis: Twenty-six years' experience. J Korean Med Sci. 23: 378-82.

$\mathrm{Al}$ Halees Z. 2007. The choice of valve prosthesis: Are the guidelines for everyone? Asian Cardiovasc \& Thorac Ann. 15(6): 457-58.

Buttard P, Bonnefoy E, Chevalier P, Marcaz PB, Robin J, Obadia JF, et al. 1997. Mechanical cardiac valve thrombosis in patients in critical hemodynamic compromise. Eur J Cardiothorac Surg. 11: 710-13. 
Choudhary SK, Talwar S, Airan B. 2016. Choice of prosthetic valve in a developing country. Heart Asia. 8(1): 65-72.

Durrleman N, Pellerin M, Bouchard D, Hebert Y, Cartier R, Perrault LP, et al. 2004. Prosthetic valve thrombosis: Twenty-year experience at the Montreal Heart Institute. J Thorac Cardiovasc Surg. 127: 1388-92.

El-Aroussy W, EL-Hagracy N, Fawzy H, Zaher S, Taha N, Moustafa S. 2013. Prevalence of rheumatic valvular heart disease among Egyptian school children: an echocardiographic screening. Med J Cairo Univ. 81(1): 139-144.

Elassy SM, Elmidany AA, Elbawab HY. 2014. Urgent cardiac surgery during pregnancy: a continuous challenge. Ann Thorac Surg. 97:1624-29.

Elsayed AA, Abdelaal KM, Abdelghaffar M, Mohamed E, Mahran T, Ahmed M, et al. 2019. Poor outcome of surgical management of acute malfunctioning mechanical mitral valve during pregnancy. Should centers with limited resources find different options? Heart Surg Forum. 22(5): E405-10.

Hassouna A, El-Ghanam M, Moftah H, Samir K, Refaat K. 2020. Index of deterioration of patients with mechanical prosthetic heart valve thrombosis. The Cardiothorac Surgeon. 28: 23-34.

Heuvelman HJ, Arabkhani B, Cornette JM, Pieper PG, Borges AJ, Takkenberg JJ, et al. 2013. Pregnancy outocmes in women with aortic valve substitutes. Am J Cardiol. 111: 382-87.

Lawley CM, Algert CS, Ford JB, Nippita TA, Figtree GA, Roberts CL. 2014. Heart valve prostheses in pregnancy: outcomes for women and their infants. J am Heart Assoc. 3: e000953.

Ma W, Hou B, Abdurusul A, Gong D, Tang Y, Chang Q, et al. 2015. Dysfunction of mechanical heart valve prosthesis: experience with surgical management in 48 patients. J Thorac Dis. 7(12); 2321-29.

Martinell J, Jimenez A, Rabago G, Artiz V, Fraile J, Farre J. 1991. Mechanical cardiac valve thrombosis. Is thrombectomy justified? Circulation. 84(5): III70-5.

Meuris B, Verbeken E, Flameng W. 2005. Mechanical valve thrombosis in a chronic animal model: differences between monoleaflet and bileaflet vavles. J Heart Valv Dis. 14(1): 96-104.

Nishimura RA, Otto CM, Bonow RO, Carabello BA, Erwin JP, Guyton RA, et al. 2014. 2014 AHA/ACC Guideline for the Management of Patients With Valvular Heart Disease. A Report of the American College of Cardiology/American Heart Association Task Force on Practice Guidelines. Circulation. 129: e521-e643.

Regitz-Zagrosek V, Roos-Hesselink JW, Bauersachs J, Blomstrom-Lundqvist C, CIfkova R, De Bonis M, et al. 2018. 2018 ESC Guidelines for the management of cardiovascular diseases during pregnancy. Eur Heart J. 39:3165-3241.

Roudaut R, Roques X, Lafitte S, Choukroun E, Laborde N,Madona F, et al. 2003. Surgery for prosthetic valve obstruction. A single center study of 136 patients. Eur J Cardiothorac Surg. 24: 868-72.

Sharaf AY, Ibrahim A, Elhamami M. 2017. Knowledge and adherence to oral anticoagulant therapy among patients with mechanical heart valve prosthesis. IOSR J Nurs \& Health Scien. 6 (3): 19-29.

Sillesen M, Hjortdal V, Vejlstrup N, Sorensen K. 2011. Pregnancy with prosthetic heart valves -30 years' nationwide experience in Denmark. Eur J Cardioth Surg. 40:448-54.

Soler-Soler J, Galve E. 2000. Worldwide perspective of valve disease. Heart. 83: 721-25.

Tsai KT, Lin PJ, Chang CH, Chu JJ, Chang JP, Kao Cl, et al. 1993. Surgical management of thrombotic disc valve. Ann Thorac Surg. 55: 98-101.

Van Hagen IM, Roos-Hesselink JW, Ruys TP, Merz WM, Goland S, Gabriel H, et al. 2015. Pregnancy in women with a mechanical heart valve: Data of the European Society of Cardiology Registry of Pregnancy and Cardiac Disease (ROPAC). Circulation. 132: 132-42.

Vause S, Clarke B, Tower CL, Hay C, Knight M. 2017. Pregnancy outcomes in women with mechanical prosthetic heart valves: A prospective descriptive population based study using the United Kingdom Obstetric Surveillance System (UKOSS) data collection system. BJOG. 124: 1411-19.

Williams MA, Crause L, Van Riet S. 2004. A comparison of mechanical valve performance in a poorly anticoagulated community. J Card Surg. 19: 410-14.

Yin W, Krukenkamp IB, Saltman AE, Gaudette G, Suresh K, Bernal O, et al. 2006. Thrombogenic performance of a St Jude bileaflet mechanical heart valve in a sheep model. ASAIO J. 52(1): 28-33. 